\title{
An MRI-defined measure of cerebral lesion severity to assess therapeutic effects in multiple sclerosis
}

\author{
Gloria Kim $^{1} \cdot$ Shahamat Tauhid $^{1} \cdot$ Sheena L. Dupuy ${ }^{1} \cdot$ Subhash Tummala $^{1} \cdot$ \\ Fariha Khalid $^{1} \cdot$ Brian C. Healy $^{1} \cdot$ Rohit Bakshi $^{1,2,3}$
}

Received: 9 September 2015/Revised: 22 December 2015/Accepted: 23 December 2015/Published online: 11 January 2016 (C) The Author(s) 2016. This article is published with open access at Springerlink.com

\begin{abstract}
Assess the sensitivity of the Magnetic Resonance Disease Severity Scale (MRDSS), based on cerebral lesions and atrophy, for treatment monitoring of glatiramer acetate (GA) in relapsing-remitting multiple sclerosis (MS). This retrospective non-randomized pilot study included patients who started daily GA $[n=23$, age (median, range) $41(26.2,53.1)$ years, Expanded Disability Status Scale (EDSS) score $1.0(0,3.5)]$, or received no disease-modifying therapy (noDMT) $[n=21$, age 44.8 $(28.2,55.4)$, EDSS $0(0,2.5)]$ for 2 years. MRDSS was the sum of $z$-scores (normalized to a reference sample) of T2 hyperintense lesion volume $(\mathrm{T} 2 \mathrm{LV})$, the ratio of $\mathrm{T} 1$ hypointense LV to T2LV (T1/T2), and brain parenchymal fraction (BPF) multiplied by negative 1 . The two groups were compared by Wilcoxon rank sum tests; within group change was assessed by Wilcoxon signed rank tests. Glatiramer acetate subjects had less progression than noDMT on T1/T2 [(median $z$-score change (range), 0 $(-1.07,1.20)$ vs. $0.41(-0.30,2.51), p=0.003)]$ and MRDSS $[0.01(-1.33,1.28)$ vs. $0.46(-1.57,2.46)$, $p=0.01]$; however, not on BPF $[0.12(-0.18,0.58)$ vs. $0.10(-1.47,0.50), p=0.59]$ and T2LV $[-0.03(-0.90$, $0.57)$ vs. $0.01(-1.69,0.34), p=0.40]$. While GA subjects
\end{abstract}

Rohit Bakshi

rbakshi@post.harvard.edu

1 Department of Neurology, Brigham and Women's Hospital, Laboratory for Neuroimaging Research, Partners MS Center, Harvard Medical School, Boston, MA, USA

2 Department of Radiology, Brigham and Women's Hospital, Laboratory for Neuroimaging Research, Partners MS Center, Harvard Medical School, Boston, MA, USA

3 Laboratory for Neuroimaging Research, One Brookline Place, Brookline, MA 02445, USA worsened only on BPF $[0.12(-0.18,0.58), p=0.001]$, noDMT worsened on BPF $[0.10(-1.47,0.50), p=0.002]$, $\mathrm{T} 1 / \mathrm{T} 2[0.41(-0.30,2.51), p=0.0002]$, and MRDSS [0.46 $(-1.57,2.46), p=0.0006]$. These preliminary findings show the potential of two new cerebral MRI metrics to track MS therapeutic response. The T1/T2, an index of the destructive potential of lesions, may provide particular sensitivity to treatment effects.

Keywords MRI - Multiple sclerosis · Glatiramer acetate · Brain atrophy $\cdot$ Composite scale

\section{Introduction}

MRI has provided a range of tools to define pathological changes in the brain and spinal cord in patients with multiple sclerosis (MS) [1]. Prior studies have combined cerebral MRI lesion and atrophy measures to create composite scales to increase sensitivity, provide a comprehensive assessment of disease status, and, in turn, provide more clinical relevance than individual MRI measures on their own [2-11]. The first such measure, the Z4 score, combined measures of disease activity and disease severity, and has been applied to assess treatment response [2, 4, 8]. These MS-MRI composite scales have shown higher effect sizes in their relationship to clinical status or longitudinal change as compared to established individual MS-MRI measures [5-7, 11, 12]. Building on this previous work, we tested a cerebral MRI composite scale focusing on MS disease severity as shown by lesions and atrophy which also includes an assessment of the destructive potential of individual lesions, the intrasubject ratio of $\mathrm{T} 1$ hypointense to T2 hyperintense lesion volume (T1/T2). Known as the Magnetic Resonance Disease Severity Scale (MRDSS) [5], 
this tool has shown higher effect sizes in differentiating relapsing-remitting (RR) from secondary progressive (SP) patients and higher longitudinal sensitivity than the component MRI measures on their own [5, 7]. To date, the role of the MRDSS in treatment monitoring has not been tested.

Glatiramer acetate (GA) is an established disease-modifying therapy (DMT) for RRMS [13-16]. GA is known to effectively reduce relapse rates and the appearance of new gadolinium (Gd)-enhancing and $\mathrm{T} 2$ hyperintense lesions [16]. In addition, GA limits the progression of destructive aspects of the disease including the rate of brain atrophy [17] and the evolution of new Gd-enhancing lesions converting to chronic T1 hypointensities ("black holes") [18]. The goal of the present pilot study was to evaluate the 2-year longitudinal sensitivity of the MRDSS in comparing GA-treated to untreated patients with RRMS. Furthermore, this study provided the unique opportunity to assess the role of $\mathrm{T} 1 / \mathrm{T} 2$ in tracking therapeutic response.

\section{Methods}

\section{Subjects}

Baseline demographic and clinical characteristics are summarized in Table 1. This was a retrospective non-randomized two-arm observational exploratory study. Given the sample size and study design, the results should be considered preliminary. All subjects were identified by chart review using the following inclusion criteria: RRMS [19], age 18-55, and an Expanded Disability Status Scale (EDSS) [20] score 0-5. Among the 44 patients with
RRMS, two groups were identified based on DMT use during the 2-year observation period: (1) GA-treated $(n=23)$ and (2) no DMT (noDMT, $n=21)$, i.e. patients who remained off DMT. Patients were required to have a baseline and 2-year brain MRI available. Clinical evaluation, including EDSS scoring [20] and timed 25 foot walk (T25FW) [21], were assessed by the treating neurologist at the Partners MS Center. When comparing groups on baseline clinical and demographic characteristics, the noDMT group showed a trend to a higher percentage of women $(p=0.07)$ and higher age $(p=0.09)$, and a significantly higher disease duration $(p=0.008)$ and lower EDSS score $(p=0.048)$. This study was approved by our institution's research ethics committee and was performed in accordance with the ethical standards laid down in the 1964 Declaration of Helsinki and its later amendments.

\section{MRI acquisition}

All patients underwent baseline and follow-up 1.5 $\mathrm{T}$ brain MRI on a fleet of Signa scanners (General Electric, Milwaukee, WI) at our institution. All scans covered the whole brain in the axial plane and included T1-weighted spinecho [repetition time and echo time (TR/TE): 550-783/ $20 \mathrm{~ms}$ ] and T2-weighted conventional spin-echo dual-echo series (TR/TE1/TE2: 2750-3000/30/80 ms), with voxel sizes of either $0.9375 \times 0.9375 \times 3 \mathrm{~mm}$ or $0.8594 \times$ $0.8594 \times 3 \mathrm{~mm}$, and no inter-slice gaps. The T1-weighted series was repeated 5-7 min after the intravenous infusion of single-dose Gd. MRI analysis was performed by observers who were unaware of the clinical details.
Table 1 Subjects'

characteristics and clinical findings at baseline and 2-year follow-up

\begin{tabular}{llll}
\hline & Glatiramer acetate & NoDMT & $p$ value \\
\hline Baseline & & & \\
Number of patients & $n=23$ & $n=21$ & - \\
Women, number (\%) & $15(65)$ & $19(90)$ & 0.07 \\
Age (years) & $41.3(26.2,53.1)$ & $44.8(28.2,55.4)$ & 0.09 \\
Disease duration (years) & $1.8(0.3,20.3)$ & $6.5(0.4,33.5)$ & 0.008 \\
EDSS score & $1.0(0,3.5)$ & $0(0,2.5)$ & 0.048 \\
T25FW (s) & $4.2(3.0,5.6)$ & $5.0(3.4,6.0)$ & 0.32 \\
Follow-up & & & \\
EDSS score & $1.0(0,3.0)$ & $0(0,2.0)$ & 0.39 \\
T25FW (s) & $4.2(2.7,6.0)$ & $4.5(3.5,5.7)$ & 0.07 \\
On-study annualized relapse rate (mean) & 0.13 & 0.12 & 0.88 \\
\hline
\end{tabular}

Key: values are median (range), unless otherwise indicated

The $p$ value for women-Fisher's exact test, for on-study relapses-Poisson regression, and the other $p$ values-Wilcoxon rank sum tests

NoDMT not receiving disease-modifying therapy, RRMS relapsing-remitting multiple sclerosis, EDSS Expanded Disability Status Scale, T25FW timed 25 foot walk

a Time since first symptoms 


\section{MRI analysis}

\section{T2 hyperintense lesion volume and whole brain atrophy}

Using the dual-echo T2-weighted images, the T2 hyperintense lesion volume (T2LV) and the normalized whole brain volume (brain parenchymal fraction-BPF) were calculated by an automated template-driven segmentation (TDS+) [22].

\section{T1 hypointense lesion volume/T2 hyperintense lesion volume ratio}

T1 hypointense lesions were initially identified and traced by a trained observer using a semi-automated edge-finding tool in Jim software (v.7; Xinapse Systems, West Bergholt, UK; http://www.xinapse.com). Each lesion and its contour was then confirmed by an experienced observer. T1 hypointense lesions were defined as appearing hypointense to the surrounding white matter, with corresponding hyperintensity on both of the dual-echo images. The lesions were also required to show non-enhancement on post-Gd images. To assess the destructive potential of lesions, the ratio of the $\mathrm{T} 1$ hypointense lesion volume to the T2LV (T1/T2) was calculated for each patient. This was used in favor of the total $\mathrm{T} 1$ hypointense lesion volume (T1LV) based on our previous work showing high co-linearity between T1LV and T2LV [5].

\section{Calculation of MRDSS}

Magnetic Resonance Disease Severity Scale was derived by calculating $z$-scores for appropriate transformations of each component as in the original paper [5], and the mean and standard deviation used in the $z$-score calculation were the values from the original paper to ensure that these were representative of a typical clinic-based MS population [5]. For the BPF, no transformation was required, but the $z-$ score was multiplied by -1 so that higher values of $-\mathrm{zBPF}$ represented lower BPF and worse disease severity. For $\mathrm{T} 2 \mathrm{LV}$, a log transformation was used, and the $z$-score was referred to as zT2LV. Finally, for the T1/T2, the logistic transformation was used, and the $z$-score was named zT1/ T2. Subjects with T1/T2 of 0 were assigned a value more extreme $(\mathrm{zT} 1 / \mathrm{T} 2=-2.5)$ than the smallest observed value $(\mathrm{zT} 1 / \mathrm{T} 2=-1.96)$. We note that all values for $\mathrm{T} 1 / \mathrm{T} 2$ and T2LV were rounded to two decimal places. To combine the $z$-scores, the following equation was used:

$\mathrm{zMRDSS}=-\mathrm{zBPF}+\mathrm{zT} 2 \mathrm{LV}+\mathrm{zT} 1 / \mathrm{T} 2$

The zMRDSS was not rescaled to lie between 0 and 10 as in the original paper because observations in the present sample would have been outside of this range. Rather, the
zMRDSS score was used for analysis. For simplicity purposes, the zMRDSS is referred to often as MRDSS throughout the remaining sections of the paper. This version of the MRDSS has been referred to as "MRDSS1" in a subsequent paper [10].

\section{Statistical analysis}

In order to compare the GA treated subjects to the untreated subjects in terms of MRI measures at baseline, year 2 and on-study changes in MRI measures, Wilcoxon rank sum tests were performed. To determine if there were within group changes in MRI measures over time, Wilcoxon signed rank tests were used. For clinical outcomes, the EDSS and T25FW in each group were compared at baseline and year 2 using a Wilcoxon rank sum test. To compare the groups on changes over time in clinical outcomes, a mixed effects ordinal logistic regression model was used. Finally, to compare the number of on-study relapses, a Poisson regression model was used. A $p<0.05$ was considered significant; a $p>0.05$ but $<0.10$ was considered a trend to significance. All statistical analysis was completed in the statistical package R (http://www.r-project.org) or Stata (version 14).

\section{Results}

\section{Baseline and follow-up MRI differences in the GA and noDMT groups}

When evaluating the MRI differences between the GA and noDMT groups at baseline and follow-up, no significant differences were found (Table 2). Only the T1/T2 difference at follow-up showed a trend towards significance (lower in the GA group).

\section{On-study changes in MRI measures in the GA and noDMT groups}

When comparing the 2-year on-study changes in MRI measures between the GA and noDMT groups (Table 3; Figs. 1, 2, 3, 4), subjects in the GA group had significantly less worsening of disease over the 2-year follow-up in terms of T1/T2 (Fig. 3) and MRDSS (Fig. 4). Considering the on-study changes within the GA cohort, a significant worsening was only seen in BPF $[0.12(-0.18,0.58)$, $p=0.001)$. However, when assessing the on-study changes within the noDMT group, statistically significant worsening was seen in BPF $[0.01(-1.47,0.50)$, $p=0.002], \mathrm{T} 1 / \mathrm{T} 2[0.41(-0.30,2.51), p=0.0002]$, and MRDSS $[0.46(-1.57,2.46), p=0.0006]$. Because the 
Table 2 MRI findings at baseline and 2-year follow-up

\begin{tabular}{lccl}
\hline & Glatiramer acetate & NoDMT & $p$ value \\
\hline Baseline & & & \\
BPF & $0.899(0.808,0.934)$ & $0.878(0.761,0.950)$ & 0.43 \\
T2LV (ml) & $2.57(0.75,9.31)$ & $3.51(1.00,28.69)$ & 0.29 \\
T1/T2 & $0.24(0.01,0.91)$ & $0.20(0,0.79)$ & 1 \\
-zBPF & $-1.24(-1.88,0.47)$ & $-0.84(-2.18,1.36)$ & 0.43 \\
zT2LV & $-0.79(-2.26,0.74)$ & $-0.42(-1.91,2.07)$ & 0.29 \\
zT1/T2 & $0.59(-1.92,3.11)$ & $0.42(-2.50,2.39)$ & 1 \\
zMRDSS & $-1.55(-4.35,3.38)$ & $-0.78(-4.15,4.28)$ & 0.46 \\
Follow-up & & & \\
BPF & $0.888(0.784,0.936)$ & $0.873(0.754,0.941)$ & 0.54 \\
T2LV (ml) & $2.52(0.62,9.34)$ & $2.83(0.84,26.67)$ & 0.35 \\
T1/T2 & $0.23(0.02,0.7)$ & $0.33(0.02,0.98)$ & 0.07 \\
-zBPF & $-1.03(-1.92,0.93)$ & $-0.74(-2.01,1.49)$ & 0.54 \\
zT2LV & $-0.82(-2.48,0.74)$ & $-0.68(-2.12,1.99)$ & 0.35 \\
zT1/T2 & $0.55(-1.41,2.04)$ & $0.91(-1.41,4.26)$ & 0.07 \\
zMRDSS & $-1.15(-3.75,2.62)$ & $-0.55(-3.53,5.64)$ & 0.16 \\
\hline Key:
\end{tabular}

Key: median (range)

All $p$ values are from Wilcoxon rank sum tests comparing the groups. The negative $\mathrm{zBPF}$ is shown so that the direction matched the others (a positive score indicates advancing disease). The MRDSS was calculated as a zMRDSS because the original scaling of the MRDSS to a $0-10$ scale led to a MRDSS of $>10$ for a follow-up score

NoDMT not receiving disease-modifying therapy, $B P F$ brain parenchymal fraction, $T 2 L V$ total cerebral $\mathrm{T} 2$ hyperintense lesion volume, T1/T2 ratio of $\mathrm{T} 1$ hypointense to $\mathrm{T} 2$ hyperintense lesion volume, MRDSS magnetic resonance disease severity scale, $z$ standardized

$p$ value for the group comparison for the difference was more statistically significant for the T1/T2 than the other individual MRI components, this implies that the difference between treatment groups in MRDSS was driven by the T1/T2 change. This was confirmed by the observations in Table 3 that there was only a limited group difference in the change for the BPF or T2LV.

\section{On-study clinical changes/relapses}

On-study and follow-up clinical changes/relapse data are shown in Table 1. When comparing the groups in terms of on-study EDSS and T25FW change, a mixed effects ordinal regression model determined that there was no significant group difference in terms of change over time ( $p=0.29$ and $p=0.35$, respectively). At follow-up, there was no difference between groups in EDSS score $(p=0.39)$. At follow-up, the T25FW trended to being lower in the GA vs. the noDMT group $(p=0.07)$. Patients in the GA group had an average of 0.26 clinical relapses over the 2 years (mean annualized attack rate $=0.13$ ). This was 0.24 (mean annualized attack rate $=0.12$ ) in the noDMT group. A Poisson regression model determined that there was no significant difference between the groups in terms of on-study relapses $(p=0.88)$.

\section{Discussion}

In this pilot study, we explored the use of a cerebral MRIbased composite scale of disease severity, based on lesions and atrophy, the MRDSS [5, 7], to assess the response to GA in RRMS over 2 years. The main findings in this study are that the two established measures of lesions and atrophy, T2LV and BPF, did not show a difference between GA and an untreated group. However, the MRDSS was sensitive to a group difference, which was driven by the scale's inclusion of a unique metric of the destructive potential of lesions, the intrasubject $\mathrm{T} 1 / \mathrm{T} 2$ ratio. These preliminary results underscore the potential limitations of conventional MRI metrics for longitudinal monitoring and show the potential utility of a more comprehensive consideration of structural changes that may relate to disease evolution, particularly the representation of lesion destructive potential.

While the present study is the first to consider the use of MRDSS or T1/T2 to monitor treatment response in MS, the

Table 3 MRI 2-year on-study changes

\begin{tabular}{lclll}
\hline & Glatiramer acetate & $p$ value & NoDMT & $p$ value \\
\hline -zBPF & $0.12(-0.18,0.58)$ & 0.001 & $0.10(-1.47,0.50)$ & 0.002 \\
zT2LV & $-0.03(-0.90,0.57)$ & 0.26 & $0.01(-1.69,0.34)$ & 0.95 \\
zT1/T2 & $0(-1.07,1.20)$ & 0.90 & $0.41(-0.30,2.51)$ & 0.0002 \\
zMRDSS & $0.01(-1.33,1.28)$ & 0.82 & $0.46(-1.57,2.46)$ & 0.0006 \\
\hline
\end{tabular}

Key: median (range)

All $p$ values are from Wilcoxon signed rank tests assessing whether there was a significant change over 2 years for each measure within each group. The negative zBPF is shown so that the direction matched the others (a positive score indicates advancing disease). The MRDSS was calculated as a zMRDSS because the original scaling of the MRDSS to a 0-10 scale led to a MRDSS of $>10$ for a follow-up score

NoDMT not receiving disease-modifying therapy, $B P F$ brain parenchymal fraction, $T 2 L V$ total cerebral T2 hyperintense lesion volume, $T 1 / T 2$ ratio of T1 hypointense to T2 hyperintense lesion volume, MRDSS magnetic resonance disease severity scale, $z$ standardized 
GA

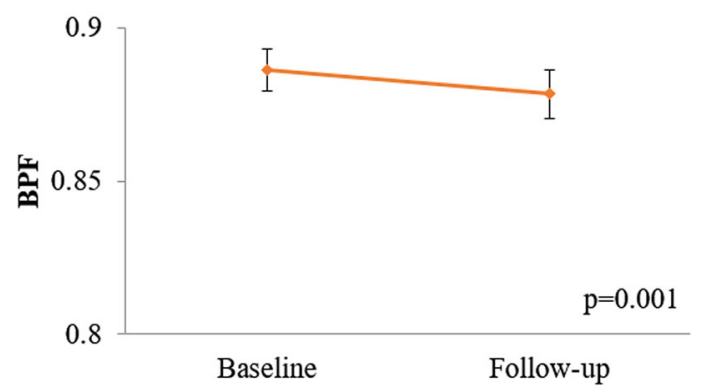

NoDMT

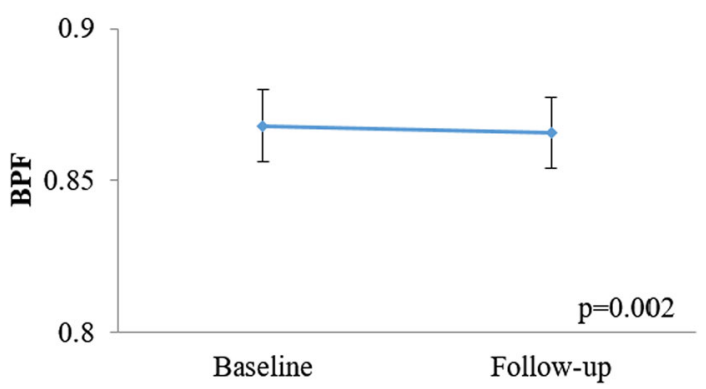

Fig. 1 On-study brain atrophy in glatiramer acetate vs. untreated patients. Mean ( \pm standard error of the mean) of brain parenchymal fraction (BPF) at baseline and 2-year follow-up. A lower score indicates advancing disease. Both glatiramer acetate (GA) and no disease modifying therapy (noDMT) cohorts showed significant decreases in BPF (i.e. no brain atrophy) from baseline to follow-up. The $p$ values in the figure are from Wilcoxon signed rank tests for the within group change over time. Furthermore, when comparing the change in zBPF between the two groups, no difference was found (Wilcoxon rank sum test, $p=0.59$ )

$\mathrm{Z} 4$ composite score has been tested in the past. In a clinical trial of roquinimex (linomide), the $\mathrm{Z} 4$ significantly distinguished the treatment groups at 3 months, and showed a similar pattern between the groups at 6 months. In a study evaluating the long term impact of GA therapy in a placebo-controlled cross-over trial, the Z4 discriminated patients well according to duration of therapy and clinical outcome [3]. In a placebo-controlled trial of anti-chlamydial therapy in RRMS, the Z4 increased in the placebo group and decreased in the antibiotic treatment group over 1 year [4]. These differences suggested a trend towards the benefit of therapy and were driven by the stabilization in the brain atrophy component of the scale. Unlike the Z4, our MRDSS uses a novel measure of lesion severity, the T1/T2 ratio, combined with overall burden of disease (T2LV) and brain atrophy (BPF).

Prior work in developing the MRDSS has shown several attractive features setting the stage for testing its sensitivity in treatment monitoring $[5,7]$. First, the MRDSS showed a higher effect size in differentiating RR vs. SP MS phenotype groups when compared to the individual component MRI measures [5]. Second, the MRDSS showed a higher
GA

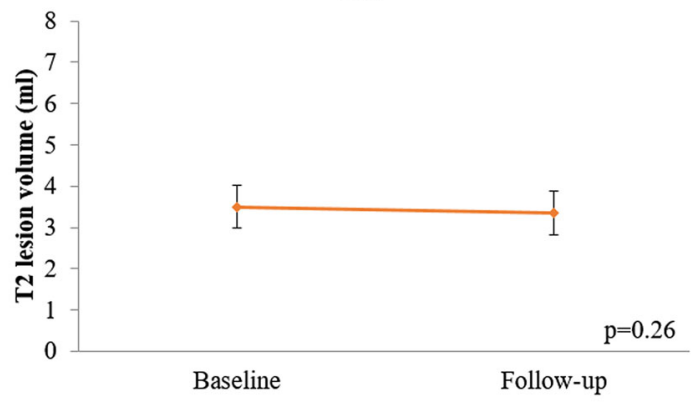

NoDMT

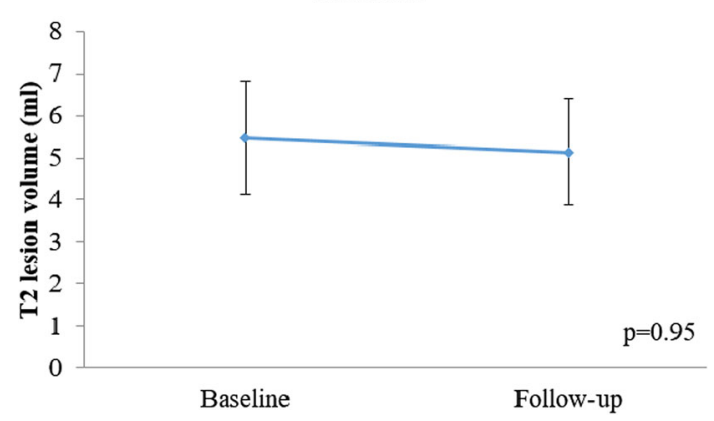

Fig. 2 On-study T2 hyperintense lesion volume changes in glatiramer acetate vs. untreated patients. Mean $( \pm$ standard error of the mean) of total cerebral T2 hyperintense lesion volume (T2LV) at baseline and 2-year follow-up. A higher score indicates advancing disease. Both glatiramer acetate (GA) and no disease modifying therapy (noDMT) cohorts showed no significant decreases from baseline to follow-up. The $p$ values in the figure are from Wilcoxon signed rank tests for the within group change over time. Furthermore, when comparing the change in zT2LV between the two groups, no difference was found (Wilcoxon rank sum test, $p=0.40$ )

correlation with physical disability than what was seen with conventional MRI lesion load [5]. Third, in a 3-year longitudinal study, the MRDSS was more sensitive to change in both RR and SP phenotype groups than the individual component MRI measures [7]. Fourth, the MRDSS showed the largest effect size in differentiating cognitively preserved vs. cognitively impaired patients with MS when compared to the individual component MRI measures [10]. Finally, the standardization of measures that is necessary to derive the MRDSS most likely improves the clinical relevance [5]. The current results extend our previous observations in that the MRDSS detected a difference between treatment groups that was not apparent when considering the established measures of conventional lesion load or brain atrophy on their own. Taken together, these exploratory findings underscore the potential advantages of a comprehensive scale of MRI-defined disease severity that considers several related but different aspects of disease pathophysiology.

The MRDSS detected a group difference that was not apparent by considering changes in either T2LV or BPF. It 
GA

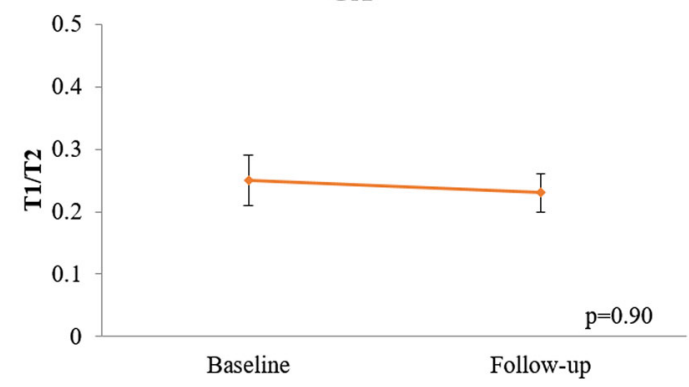

NoDMT

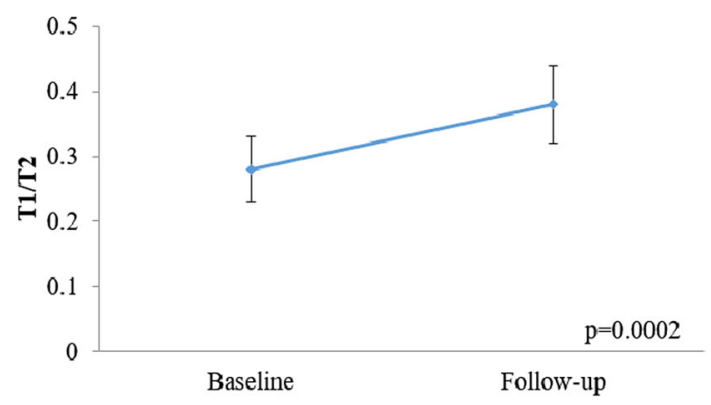

Fig. 3 On-study T1/T2 ratio changes in glatiramer acetate vs. untreated patients. Mean ( \pm standard error of the mean) of the ratio of total cerebral $\mathrm{T} 1$ hypointense to $\mathrm{T} 2$ hyperintense lesion volume (T1/T2) at baseline and 2-year follow-up. A higher score indicates advancing disease. The no disease modifying therapy (noDMT) group showed significant worsening, but the glatiramer acetate (GA) treated group did not. The $p$ values in the figure are from Wilcoxon signed rank tests for the within group change over time. Furthermore, when comparing the change in $\mathrm{zT} 1 / \mathrm{T} 2$ between groups, a difference was found favoring GA treatment (Wilcoxon rank sum test, $p=0.003$ )

was clear from examination of the data, that the group difference in MRDSS, suggesting a treatment effect of GA, was dominated by changes in the T1/T2. This is the intrasubject ratio of the volume of cerebral $\mathrm{T} 1$ hypointense to $\mathrm{T} 2$ hyperintense lesions. This ratio roughly equates to an index of the destructive potential of a patient's lesions in that it is well known that chronic T1 hypointensity indicates severe destructive pathology (severe irreversible demyelination and axonal loss) [23-25]. Given that the scale is derived from a single time point scan, the chronicity of $\mathrm{T} 1$ hypointensity is not assured. But, the exclusion of Gd-enhancing lesions from our definition of T1 hypointense lesions reduced the likelihood of including transient/benign T1 hypointense lesions [26]. Furthermore, the high clinical relevance of the T1/T2 has been suggested by several previous studies. One study showed a higher T1/ $\mathrm{T} 2$ in SP vs. RR MS, with higher effect sizes than T2LV or BPF differences [5]. In a 3-year longitudinal study, the T1/ T2 was more sensitive to change in both RR and SP phenotype groups than T2LV or BPF [7]. In addition, the T1/ $\mathrm{T} 2$ showed a larger effect size in differentiating cognitively preserved vs. cognitively impaired patients with MS when
GA

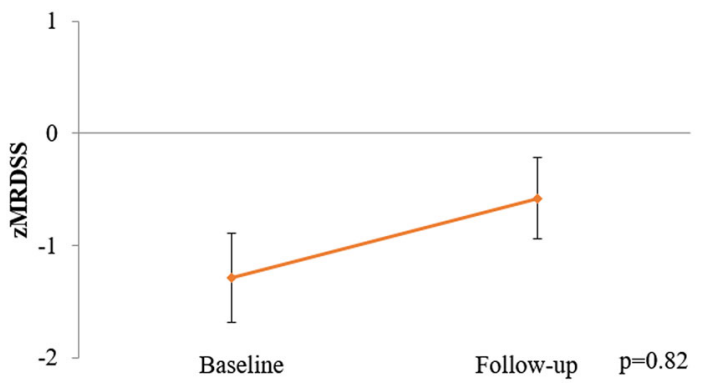

NoDMT

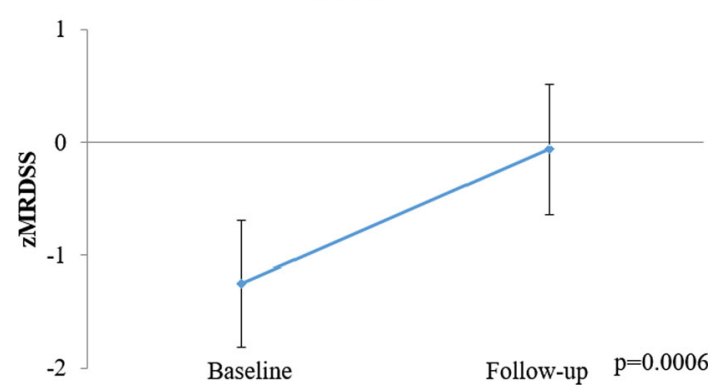

Fig. 4 On-study MRDSS changes in glatiramer acetate vs. untreated patients. Mean ( \pm standard error of the mean) of the magnetic resonance disease severity scale (zMRDSS) at baseline and 2-year follow-up. A higher score indicates advancing disease. The no disease modifying therapy (noDMT) group showed significant worsening, but the glatiramer acetate (GA) treated group did not. The $p$ values in the figure are from Wilcoxon signed rank tests for the within group change over time. Furthermore, when comparing the change in zMRDSS between groups, a difference was found favoring GA treatment (Wilcoxon rank sum test, $p=0.01$ )

compared to T2LV [10]. The T1/T2 shows only moderate correlations with either T2LV or BPF [5], indicating its ability to detect divergent aspects of disease severity. The pathobiologic factors contributing to the tendency towards more destructive lesions in MS patients are unknown. One line of investigation relates to genetic predisposition [27, 28]. There is a relatively low co-linearity between $\mathrm{T} 1 / \mathrm{T} 2$ and either T1LV, T2LV, or BPF [5]. Taken together, these observations suggest the unique role of the information provided by $\mathrm{T} 1 / \mathrm{T} 2$ that may complement the evaluation of cerebral disease severity obtained by standard evaluations of lesion load and atrophy.

There are several lines of evidence regarding the effect of GA therapy in MS that may help to explain the selective effect on T1 hypointense-associated lesion characteristics suggested in this pilot study. While most of the available DMTs for MS act by reducing lymphocyte entry into the CNS, GA is thought to have a unique mechanism of action $[29,30]$. Animal model and clinical studies show the effect of GA on shifting pro-inflammatory to anti-inflammatory immune actions [29, 30]. In addition to immunomodulatory effects, the drug may also promote the secretion of 
neurotrophins to enhance repair processes and remyelination [29]. Consistent with this hypothesis, a placebo-controlled phase III clinical trial showed that the percentage of new cerebral lesions evolving into chronic T1 hypointensities was lower in GA-treated vs. placebo-treated RRMS patients [18]. Such a treatment effect of GA has been confirmed in subsequent studies of different patient populations [31, 32]. In the present study, when comparing the change in zT1/T2 between groups, a significant difference was found favoring GA treatment vs. no treatment. This was the result of a significant increase in the T1/T2 in untreated patients but no change in GA patients over 2 years. Taken together, while preliminary and requiring confirmation in larger studies, these results suggest that GA can limit tissue destruction in lesions once they have formed leading to a reduced level of long term tissue injury.

In the present study, the GA and untreated groups did not differ on their rates of changes in T2LV or BPF over the 2-year observation period. This is in contrast to the results of large phase III placebo-controlled studies, in which GA reduced the rate of progression of T2LV in RRMS [14] or clinically-isolated demyelinating syndromes [33]. Our study, given the much smaller sample size and retrospective study design, may have been under-powered to show such effects. Regarding the effect of GA on limiting brain atrophy, results have been inconsistent [34], showing either a partial delayed effect [17, $35]$ or no effect $[33,36]$ in the above-referenced phase III studies. Furthermore, the sensitivity of brain atrophy as a longitudinal monitoring tool in the evaluation of MS therapies has been hampered by several factors [37, 38]. The limitations include the delayed effect of newly started DMT on atrophy (usually requiring a lag time of several months to a year), the partial effect (a maximum benefit of up to $40-50 \%$ per year in the rate of reduction), and the confounding effects on brain volume of acute DMT-related or corticosteroid brain volume change (i.e. pseudoatrophy due to anti-inflammatory effects and fluid shifts) [37, 38]. Furthermore, other unexpected factors may alter brain volume measurements such as diurnal fluctuations [39]. Taken together, the above observations suggest it is perhaps not surprising that our study failed to detect any difference in T2LV or BPF change between the two groups in this small study.

Our study was not without several limitations. This work was exploratory and the findings should be considered preliminary. The "real world" subject groups may have been biased due to the retrospective study design and non-randomized treatment assignment. The groups were not ideally matched at study entry on male/female ratio, age, disease duration, and disability. This may have affected the results in that, for example, the higher disability, higher percentage of men, and lower disease duration at entry in the GA group may have led to a bias. Future studies should be properly designed to provide more definitive results which would allow extension and confirmation of our observations. Posthoc analyses of phase III trial data would be particularly helpful to overcome several of these limitations. Advanced MRI techniques may prove more specific for use at a single time point in the identification of the most destructive lesions rather than relying on the volume of hypointensity on T1weighted images [26, 40]. Finally, to better understand the full breadth of any potential treatment effects of GA, it would be of interest to incorporate MRI measures of gray matter and spinal cord pathology. Both of these aspects of disease severity have shown a benefit in improving the validity of MS-MRI composite scales [10, 11].

Acknowledgments We thank the staff of the CLIMB study at the Partners MS Center for valuable collaboration.

\section{Compliance with ethical standards}

Conflicts of interest This study was supported in part by a research grant from Teva Neuroscience, Inc. A Medical Accuracy Review of this manuscript was conducted by Teva. We thank Mariann PolgarTurcsanyi for database management and Mark C. Anderson for technical assistance. Dr. Healy received research support from MerckSerono, Genzyme, and Novartis. Dr. Bakshi received consulting fees from AbbVie, Alkermes, Biogen, Novartis, and Questcor and research support from Biogen, Merck-Serono, Novartis, Genzyme and Teva. The other authors have nothing to disclose.

Open Access This article is distributed under the terms of the Creative Commons Attribution 4.0 International License (http://creative commons.org/licenses/by/4.0/), which permits unrestricted use, distribution, and reproduction in any medium, provided you give appropriate credit to the original author(s) and the source, provide a link to the Creative Commons license, and indicate if changes were made.

\section{References}

1. Bakshi R, Thompson AJ, Rocca MA, Pelletier D, Dousset V, Barkhof $\mathrm{F}$ et al (2008) MRI in multiple sclerosis: current status and future prospects. Lancet Neurol 7:615-625

2. Wolinsky JS, Narayana PA, Noseworthy JH, Lublin FD, Whitaker JN, Linde A et al (2000) Linomide in relapsing and secondary progressive MS: part II: MRI results. Neurology 54:1734-1741

3. Wolinsky JS, Narayana PA, Johnson KP, Multiple Sclerosis Study Group and the MRI Analysis Center (2001) United States open-label glatiramer acetate extension trial for relapsing multiple sclerosis: MRI and clinical correlates. Mult Scler 7:33-41

4. Sriram S, Yao SY, Stratton C, Moses H, Narayana PA, Wolinsky JS (2005) Pilot study to examine the effect of antibiotic therapy on MRI outcomes in RRMS. J Neurol Sci 234:87-91

5. Bakshi R, Neema M, Healy BC, Liptak Z, Betensky RA, Buckle GJ et al (2008) Predicting clinical progression in multiple sclerosis with the magnetic resonance disease severity scale. Arch Neurol 65:1449-1453

6. Poonawalla AH, Datta S, Juneja V, Nelson F, Wolinsky JS, Cutter G et al (2010) Composite MRI scores improve correlation with EDSS in multiple sclerosis. Mult Scler 16:1117-1125

7. Moodie J, Healy BC, Buckle GJ, Gauthier SA, Glanz BI, Arora A et al (2012) Magnetic resonance disease severity scale (MRDSS) for patients with multiple sclerosis: a longitudinal study. J Neurol Sci 315:49-54 
8. Wolinsky JS, Narayana PA, Nelson F, Datta S, O'Connor P, Confavreux C et al (2013) Magnetic resonance imaging outcomes from a phase III trial of teriflunomide. Mult Scler 19:1310-1319

9. Kosa P, Komori M, Waters R, Wuemail T, Corteseemail I, Ohayonemail J et al (2015) Novel composite MRI scale correlates highly with disability in multiple sclerosis patients. Mult Scler Relat Disord 4:526-535

10. Bakshi R, Neema M, Tauhid S, Healy BC, Glanz BI, Kim G et al (2014) An expanded composite scale of MRI-defined disease severity in multiple sclerosis: MRDSS2. NeuroReport 25:1156-1161

11. Nelson F, Poonawalla AH, Datta S, Banuelos RC, Rahbar MH et al (2015) Association of multiple sclerosis related cognitive impairment with an MRI-derived composite score. J Mult Scler 2:1

12. Mainero C, De Stefano N, Iannucci G, Sormani MP, Guidi L, Federico A et al (2001) Correlates of MS disability assessed in vivo using aggregates of MR quantities. Neurology 56:1331-1334

13. Johnson KP, Brooks BR, Cohen JA, Ford CC, Goldstein J, Lisak $\mathrm{RP}$ et al (1995) Copolymer 1 reduces relapse rate and improves disability in relapsing-remitting multiple sclerosis: results of a phase III multicenter, double-blind placebo-controlled trial. Neurology 45:1268-1276

14. Comi G, Filippi M, Wolinsky JS (2001) European/Canadian multicenter, double-blind, randomized, placebo-controlled study of the effects of glatiramer acetate on magnetic resonance imaging-measured disease activity and burden in patients with relapsing multiple sclerosis. Ann Neurol 49:290-297

15. Ford C, Goodman AD, Johnson K, Kachuck N, Lindsey JW, Lisak R et al (2010) Continuous long-term immunomodulatory therapy in relapsing multiple sclerosis: results from the 15 -year analysis of the US prospective open-label study of glatiramer acetate. Mult Scler 16:342-350

16. Scott LJ (2013) Glatiramer acetate: a review of its use in patients with relapse-remitting multiple sclerosis and in delaying the onset of clinically definite multiple sclerosis. CNS Drugs 27:971-988

17. Sormani MP, Rovaris M, Valsasina P, Wolinsky JS, Comi G, Filippi M (2004) Measurement error of two different techniques for brain atrophy assessment in multiple sclerosis. Neurology 62:1432-1434

18. Filippi M, Rovaris M, Rocca MA, Sormani MP, Wolinsky JS, Comi G et al (2001) Glatiramer acetate reduces the proportion of new MS lesions evolving into 'black holes'. Neurology 57:731-733

19. Polman CH, Reingold SC, Edan G, Filippi M, Hartung HP, Kappos L et al (2005) Diagnostic criteria for multiple sclerosis: 2005 revisions to the "McDonald Criteria". Ann Neurol 58:840-846

20. Kurtzke JF (1983) Rating neurologic impairment in multiple sclerosis: an expanded disability status scale (EDSS). Neurology 33:1444-1452

21. Kaufman M, Moyer D, Norton J (2000) The significant change for the timed 25-foot walk in the multiple sclerosis functional composite. Mult Scler 6:286-290

22. Wei X, Warfield SK, Zou KH, Wu Y, Li X, Guimond A et al (2002) Quantitative analysis of MRI signal abnormalities of brain white matter with high reproducibility and accuracy. J Magn Reson Imaging 15:203-209

23. Truyen $\mathrm{L}$, van Waesberghe JH, van Walderveen MA, van Oosten BW, Polman CH, Hommes OR et al (1996) Accumulation of hypointense lesions ("black holes") on T1 spin-echo MRI correlates with disease progression in multiple sclerosis. Neurology 47:1469-1476

24. van Walderveen MA, Kamphorst W, Scheltens P, van Waesberghe JH, Ravid R, Valk J et al (1998) Histopathologic correlate of hypointense lesions on T1-weighted spin-echo MRI in multiple sclerosis. Neurology 50:1282-1288
25. Giorgio A, Stromillo ML, Bartolozzi ML, Rossi F, Battaglini M, De Leucio A et al (2014) Relevance of hypointense brain MRI lesions for long-term worsening of clinical disability in relapsing multiple sclerosis. Mult Scler 20:214-219

26. van Waesberghe JH van Walderveen MA, Castelijns JA, Scheltens P, Lycklama à Nijeholt GJ, Polman CH et al (1998) Patterns of lesion development in multiple sclerosis: longitudinal observations with T1-weighted spin-echo and magnetization transfer MR. AJNR Am J Neuroradiol 19:675-683

27. Enzinger C, Ropele S, Smith S, Strasser-Fuchs S, Poltrum B, Schmidt $\mathrm{H}$ et al (2004) Accelerated evolution of brain atrophy and "black holes" in MS patients with APOE-epsilon 4. Ann Neurol 55:563-569

28. van Veen T, Nielsen J, Berkhof J, Barkhof F, Kamphorst W, Bö L et al (2007) CCL5 and CCR5 genotypes modify clinical, radiological and pathological features of multiple sclerosis. J Neuroimmunol 190:157-164

29. Liblau R (2009) Glatiramer acetate for the treatment of multiple sclerosis: evidence for a dual anti-inflammatory and neuroprotective role. J Neurol Sci 287(Suppl 1):S17-S23

30. Aharoni R (2014) Immunomodulation neuroprotection and remyelination - the fundamental therapeutic effects of glatiramer acetate: a critical review. J Autoimmun 54:81-92

31. Zivadinov R, Dwyer M, Barkay H, Steinerman JR, Knappertz V, Khan O (2015) Effect of glatiramer acetate three-times weekly on the evolution of new, active multiple sclerosis lesions into T1hypointense "black holes": a post hoc magnetic resonance imaging analysis. J Neurol 262:648-653

32. Zivadinov R, Dwyer M, Ramasamy DP, Davis MD, Steinerman JR, Khan O (2015) The effect of three times a week glatiramer acetate on cerebral $\mathrm{T} 1$ hypointense lesions in relapsing-remitting multiple sclerosis. J Neuroimaging 25:989-995

33. Comi G, Martinelli V, Rodegher M, Moiola L, Bajenaru O, Carra A et al (2009) Effect of glatiramer acetate on conversion to clinically definite multiple sclerosis in patients with clinically isolated syndrome (PreCISe study): a randomised, double-blind, placebo-controlled trial. Lancet 374:1503-1511

34. Rovaris M, Comi G, Filippi M (2005) Can glatiramer acetate reduce brain atrophy development in multiple sclerosis? J Neurol Sci 233:139-143

35. Comi G, Martinelli V, Rodegher M, Moiola L, Leocani L, Bajenaru O et al (2013) Effects of early treatment with glatiramer acetate in patients with clinically isolated syndrome. Mult Scler 19:1074-1083

36. Rovaris M, Comi G, Rocca MA, Wolinsky JS, Filippi M, European/Canadian Glatiramer Acetate Study Group (2001) Shortterm brain volume change in relapsing-remitting multiple sclerosis: effect of glatiramer acetate and implications. European/ Canadian Glatiramer Acetate Study Group. Brain 124:1803-1812

37. Bermel RA, Bakshi R (2006) The measurement and clinical relevance of brain atrophy in multiple sclerosis. Lancet Neurol 5:158-170

38. Tsivgoulis G, Katsanos AH, Grigoriadis N, Hadjigeorgiou GM, Heliopoulos I, Kilidireas C et al (2015) The effect of disease modifying therapies on brain atrophy in patients with relapsingremitting multiple sclerosis: a systematic review and meta-analysis. PLoS ONE 10:e0116511

39. Nakamura K, Brown RA, Narayanan S, Collins DL, Arnold DL, Alzheimer's Disease Neuroimaging Initiative (2015) Diurnal fluctuations in brain volume: statistical analyses of MRI from large populations. Neuroimage 118:126-132

40. Zhang Y, Traboulsee A, Zhao Y, Metz LM, Li DK (2011) Texture analysis differentiates persistent and transient $\mathrm{T} 1$ black holes at acute onset in multiple sclerosis: a preliminary study. Mult Scler 17:532-540 